\title{
Giving Patients' Preferences a Voice in Medical Treatment Life Cycle: The PREFER Public-Private Project
}

\author{
Esther W. de Bekker-Grob ${ }^{1,2} \cdot$ Conny Berlin ${ }^{3} \cdot$ Bennett Levitan $^{4} \cdot$ Karim Raza $^{5,6}$ • \\ Kalliopi Christoforidi ${ }^{7} \cdot$ Irina Cleemput $^{8} \cdot$ Jana Pelouchova $^{9} \cdot$ Harald Enzmann ${ }^{10}$. \\ Nigel Cook $^{3}$ - Mats G. Hansson ${ }^{11}$
}

Published online: 28 February 2017

(c) Springer International Publishing Switzerland 2017

The patient perspective is important in all medical research, particularly in developing new treatments (i.e., drugs, medical devices, and vaccines). Treatments are developed for patients, and there is an emerging consensus that patients should be involved at crucial decision points in the treatment life cycle. As such, taking into consideration the patient voice has become increasingly important not only for the companies that develop new treatments but

Esther W. de Bekker-Grob

debekker-grob@bmg.eur.nl

1 Section of Health Technology Assessment and Erasmus Choice Modelling Centre, Institute of Health Policy and Management, Erasmus University Rotterdam, P.O. Box 1738, 3000 DR Rotterdam, The Netherlands

2 Section of Medical Decision Making and Erasmus Choice Modelling Centre, Department of Public Health, Erasmus MC, University Medical Centre Rotterdam, Rotterdam, The Netherlands

3 Novartis Pharma AG, Basel, Switzerland

4 Janssen R\&D LLC, Titusville, NJ, USA

5 Rheumatology Research Group, Institute of Inflammation and Ageing, University of Birmingham, Birmingham, UK

6 Sandwell and West Birmingham Hospitals NHS Trust, Birmingham, UK

7 European Cancer Patient Coalition, Brussels, Belgium

8 Belgian Health Care Knowledge Centre, Brussels, Belgium

9 Diagnóza Leukemie, Prague, Czech Republic

10 Federal Institute for Drugs and Medical Devices, Bonn, Germany

11 Department of Public Health and Caring Sciences, Centre for Research Ethics and Bioethics, Uppsala University, Uppsala, Sweden also for the authorities that assess, regulate, and decide which treatments are effective, safe, well-tolerated, and cost effective [1, 2].

In general, stakeholders (i.e., industry, regulatory authorities, health technology assessment [HTA] bodies, reimbursement agencies, clinicians, and patient organizations) all agree about the importance of incorporating patients' preferences, ${ }^{1}$ needs, and perspectives into decision making and the need to provide more avenues for patient engagement. However, there is little guidance on incorporating scientifically valid preference measurements into the treatment development life cycle or into regulatory and reimbursement decision-making processes regarding medical treatments. Important questions include the following: What is an appropriate structured approach to assess and use patient preferences during the development, approval, and post-approval phases of medical products? What kind of qualitative and quantitative methods exist to obtain insight into patient preferences? What level of validity, representativeness, and robustness is necessary? Which preference-measurement method should be used in what key decision points in the medicinal product life cycle? How will these patient preference approaches satisfy the needs of the different stakeholders, specifically regulatory, HTA, and reimbursement bodies, and feed into their existing decision-making processes? To what extent can we identify generic approaches to preference elicitation as opposed to disease- or disease area-specific approaches? How transferable are patient-preference data from country to country?

\footnotetext{
${ }^{1}$ Roughly, preferences reflect patients' values and characterize the relative importance that patients associate with the expected benefits, possible harms, and other aspects of treatment [3].
} 
The answers to these questions should accommodate the requirements of different stakeholders and decision makers in a medicine's life cycle. Therefore, combining a multidisciplinary approach with a consortium of various stakeholders is essential, allowing these urgent and relevant questions to be answered and giving patients' preferences appropriate roles in the treatment life cycle. PREFER (Patient Preferences in Benefit and Risk Assessments during the Treatment Life Cycle) is a public-private research initiative that has recently been launched to tackle these challenges [4].

\section{PREFER: What is it?}

PREFER is a 5-year research project funded equally by the Innovative Medicines Initiative (IMI; Europe's largest public-private initiative aiming to speed the development of better and safer medicines for patients) and by industry as in-kind contributions. IMI is a partnership between the EU's Horizon 2020 program and the European pharmaceutical industry represented by the European Federation of Pharmaceutical Industries and Associations (EFPIA). The PREFER consortium includes 33 partners: ten academic institutions from different European countries, 16 pharmaceutical companies from the USA and Europe, four national and international patient organizations, one HTA body, and two small- and medium-sized enterprises, all adding their experience and perspectives to the project.

PREFER builds upon the experiences and outcomes of previous initiatives, e.g., from the US FDA, European Medicines Agency (EMA), previous IMI projects such as PROTECT (Pharmacoepidemiological Research on Outcomes of Therapeutics), EUPATI (European Patients Academy on Therapeutic Innovation), and the MDIC (Medical Device Innovation Consortium) [5-9]. PREFER takes a broader approach than these initiatives, engaging competencies and perspectives from a wide set of stakeholders. It is focused on formal requirements assessment from the perspective of each stakeholder group, case studies to inform practical recommendations on when and how to perform studies to elicit patient preferences, and how these can address the requirements of and inform regulatory authorities, pharmaceutical companies, HTA bodies, and reimbursement agencies.

\section{PREFER: What are the Aims, Objectives, and Deliverables?}

The main aim of PREFER is to strengthen patient-centric decision making throughout the life cycle of medicinal treatments by developing expert and evidence-based recommendations on how patient preferences should be assessed and inform decision making. To reach this main aim, PREFER is divided into three parts, each having its own general objectives.

Part A ('Patient preferences: why, when, and how [i.e., methodology]') will first obtain insights from all stakeholders regarding their key needs, expectations, desires, and concerns about the assessment and use of patient preferences in medical decision processes. Hereto a scope literature review, in-depth interviews, and focus groups will be performed with all stakeholders from different countries until data saturation and validation has been reached. Second, it will identify and appraise qualitative and quantitative methods for preference elicitation, considering also educational and psychological tools (including serious games) that can be integrated into preferenceelicitation methods and understanding the drivers of preferences. Systematic reviews in combination with input from international health preference experts will be used to reach consensus about which methods for what key decisions are suitable to be tested in Part B.

Part B ('Testing preference-elicitation methods in clinical case studies') will use methods developed and identified in Part A to (1) test and evaluate several methods for preference elicitation in clinical case studies by focusing on different decision points in the treatment development and approval process, covering a range of diseases (common to uncommon), age groups affected, taking into account the cultural and sociological diversity within the EU, and (2) conduct computer simulation studies to both contribute to smarter design of the case studies and explore the sensitivity of preference studies. These case studies and simulations will be designed to address issues raised in the requirements-gathering step in part $\mathrm{A}$. The outputs will be evaluated together with stakeholders to ensure a 'fit' with their decision-making processes.

Part C ('Developing recommendations') will (1) generate recommendations on patient-preference elicitation to inform decision making during the medical treatment life cycle using the results from Part A and B of the PREFER project and (2) support the development of guidelines for the design, conduct, analysis, and reporting of patientpreference studies.

\section{PREFER: Why is it Necessary?}

Over the last decade, the patient voice has become heard more often in, for example, research funding bodies, institutional review boards, technology appraisal committees, regulatory and HTA body assessment panels, reimbursement decision makers, and the development of outcome measures. Although some pharmaceutical 
companies perform patient-preference research alongside their early development and registration studies, explicitly and deliberatively taking account of patient preferences is uncommon along the medicinal product life cycle. Moreover, this patient-preference research (qualitative and quantitative) may be used for internal company decision making but is not always shared with the regulatory and HTA/reimbursement bodies or even published in peer-reviewed journals. For patient-preference studies that are used within the medical treatment life cycle, standards on the design, conduct, analysis, and use of the findings in decision making are lacking. This is critical, since conducting patient preference-elicitation studies is often time consuming, expensive, and may be burdensome to patients. Additionally, while one division of one regulatory agency, FDA Centre for Devices and Radiological Health, has issued some guidance on using patient preferences to support medical device applications, most industry, regulatory authorities, HTA bodies, and reimbursement agencies have key uncertainties regarding the validity, representativeness, and robustness of preference studies to inform deliberative decision making. What is currently missing is a shared understanding among all key stakeholders of (1) what constitutes a methodologically sound patient-preference study; (2) how the results from such a study can be incorporated in the decision-making processes of industry, regulatory authorities, HTA bodies, and reimbursement agencies; and (3) at which stage(s) in the product development process and life cycle this information can best be collected. Another current gap is the lack of information on how patient-preference elicitation should be conducted in different patient populations, with acute and chronic diseases, and with more common as well as less common diseases. The recommendations that will be developed in PREFER are intended to address these outstanding questions. These recommendations will likely result in improved adaptation of medical treatments to patients' needs and wishes, which in turn will benefit satisfaction and health outcomes. A strong joint interest in the topic of this project and in developing recommendations with broad acceptance has led numerous stakeholders to provide contributions to this project via patients', regulatory, HTA/reimbursement, and scientific advisory groups.

\section{PREFER: What are the Ambitions and Impact?}

A broad array of (combinations of) patient-preference methods will be tested prospectively in several empirical and simulation case studies. The availability of large patient cohorts will enable us to test new methods or deviations from existing methods in a randomized manner by comparing well-known methods and newer ones. Based on discussions with stakeholders, suitable methods will be tested and their contributions to inform decision making will be discussed in recommendations adapted to the needs of all relevant stakeholders. The recommendations from PREFER are expected to lead to changes in practices, in that stakeholders will systematically consider whether a preference study would add value at key decision points in the medical treatment life cycle; if so, PREFER recommendations would be available to follow. Patients and patient organizations may become more involved in decision making at key decision points and develop closer relationships with industry, regulatory authorities, HTA bodies, and reimbursement agencies. Ideally, these bodies will systematically consider including the patient's perspective in their decision-making processes. The output will not only be used by these decision makers but will also serve as an additional source of information to adjust or discontinue medical treatment development processes and inform healthcare providers and patients. Some particular insights expected are guidelines for identification of subgroups of patients with distinct preferences, an understanding of the existence of cultural and economic-based differences in preferences, and support for the communication of preferences for relative benefits and risks of medical treatment-all of which will help foster the implementation of patient-centered medicine.

In summary, the ambition of PREFER is to develop a systematic approach for considering the use of patient preferences across the medical treatment life cycle. The strongest demonstration of the value of PREFER will come from acceptance of its recommendations by all stakeholders. The ideal achievement of the PREFER project would be a global, harmonized approach to the use of patientpreference studies by industry, regulatory authorities, HTA bodies, and reimbursement agencies. The horizon after the PREFER project is a world where collecting evidence on patient preferences about the relative benefits and risks of medical treatments is considered systematically alongside traditional evidence types such as efficacy, safety/adverse events, quality of life, and economic evidence.

Acknowledgements The PREFER project has received funding from the IMI 2 Joint Undertaking under grant agreement no. 115966. This joint undertaking receives support from the EU's Horizon 2020 research and innovation program and EFPIA.

\section{Compliance with Ethical Standards}

Conflict of interest Conny Berlin and Nigel Cook are employed by, own stock in, and have stock options in Novartis Pharma AG, which is one of the industry partners in the PREFER Project. Karim Raza has received consulting fees/honoraria from Janssen, Roche, Pfizer, and BMS and research grants from Pfizer and Abbvie. Esther W. de Bekker-Grob, Kalliopi Christoforidi, Irina Cleemput, Jana Pelouchova, Harald Enzmann, and Mats G. Hansson have no conflicts of 
interest that are directly relevant to the content of this article. Dr. Levitan is an employee of Janssen Research and Development, LLC. $\mathrm{He}$ is a stockholder in Johnson \& Johnson, Baxter International, Inc., Pharmaceutical Holdrs Trust, and Zimmer Holdings, Inc. He also owns stock in a variety of companies that at times include other pharmaceutical and health care-related companies.

Disclaimer This text and its contents reflects the PREFER project's view and not the view of the IMI, the EU, EFPIA, or the authors' respective organization(s).

\section{References}

1. Hoos A, Anderson J, Boutin M, et al. Partnering with patients in the development and lifecycle of medicines: a call for action. Ther Innov Regul Sci. 2015;49:929-39.

2. Smith MY, Hammad TA, Metcalf M, et al. Patient engagement at a tipping point - the need for cultural change across patient, sponsor, and regulator stakeholders: insights from the DIA conference, "Patient Engagement in Benefit Risk Assessment Throughout the Life Cycle of Medical Products". Ther Innov Regul Sci. 2016;50:546-53.

3. Medical Device Innovation Consortium (MDIC) Patient-Centered Benefit-Risk Project report. http://mdic.org/wp-content/uploads/
2015/05/MDIC_PCBR_Framework_Web.pdf. Accessed 20 Feb 2017.

4. The IMI PREFER project. http://www.imi-prefer.eu/. Accessed 20 Feb 2017.

5. US Department of Health and Human Services. Food and Drug Administration. Center for Devices and Radiological Health. Center for Biologics Evaluation and Research. Patient Preference Information-Voluntary Submission, Review in Premarket Approval Applications, Humanitarian Device Exemption Applications, and De Novo Requests, and Inclusion in Decision Summaries and Device Labelling. Guidance for industry, food and Drug Administration Staff, and Other Stakeholders. 24 August 2016. http://www.fda.gov/downloads/MedicalDevices/Device RegulationandGuidance/GuidanceDocuments/UCM446680.pdf. Accessed 20 Feb 2017.

6. Overview of EMA's interaction with patients and consumers during 2015. http://www.ema.europa.eu/docs/en_GB/document_library/ Presentation/2016/02/WC500200850.pdf. Accessed 20 Feb 2017.

7. PROTECT: Pharmacoepidemiological Research on Outcomes of Therapeutics by a European Consortium. http://protectbenefitrisk. eu/. Accessed 20 Feb 2017.

8. European Patients' Academy. http://www.patientsacademy.eu/. Accessed 20 Feb 2017.

9. The Medical Device Innovation Consortium's Patient-focused Benefit-Risk Framework. http://mdic.org/pcbr/. 